\title{
Desenvolvimento de um Chatbot para Compartilhamento e Disseminação de Informações do Tempo
}

\author{
Ana Katarine F. S. Santos ${ }^{1}$, Jen H. Liu ${ }^{2}$, Tamires P. Silva ${ }^{2}$, Ricardo A. C. Souza ${ }^{1,3}$ \\ ${ }^{1}$ Programa de Pós-graduação em Informática Aplicada (PPGIA) - Universidade Federal \\ Rural de Pernambuco - (UFRPE) Rua Dom Manoel de Medeiros s/n - Dois Irmãos - \\ 52.171-900 - Recife - PE - Brasil \\ ${ }^{2}$ Bacharelado em Ciência da Computação (BCC) - Universidade Federal Rural de \\ Pernambuco - (UFRPE) - Recife - PE - Brasil \\ ${ }^{3}$ Departamento de Computação (DC) - Universidade Federal Rural de Pernambuco \\ (UFRPE) - Recife, PE - Brasil \\ \{anakatarines, liujenhorng, tamirespereira6\}@gmail.com, \\ ricardo.souzadufrpe.br
}

Abstract. Weather conditions have a great impact on people's lives. Public organizations responsible for monitoring and disseminating weather and climate information often use technical language that can be confusing and lead to misunderstanding by non-specialist users. This paper seeks to mitigate this problem by applying a knowledge management approach called the KM Cycle to develop a solution to improve communication and dissemination of time information to ordinary people. As a result we developed a chatbot prototype integrated to different communication channels, such as Web Site and social network.

Resumo. As condições climáticas causam grande impacto na vida das pessoas. As organizações públicas responsáveis pelo monitoramento e divulgação das informações do tempo e clima geralmente utilizam uma linguagem técnica que pode se tornar confusa e levar a um mal entendimento por parte de usuários não especializados. Este trabalho procura mitigar este problema a partir da aplicação de uma abordagem de gestão do conhecimento, denominada Ciclo KM, para desenvolvimento de uma solução que visa melhorar a comunicação $e$ disseminação de informações do tempo para pessoas comuns. O resultado foi o desenvolvimento de um protótipo de chatbot integrável a diferentes canais de comunicação, como Web Site e rede social.

\section{Introdução}

As mudanças climáticas podem acarretar uma série de problemas que causam transtornos tais como dificuldade de locomoção nas cidades ou até desastres com perdas materiais e de vida humanas. Diante desse cenário houve um crescimento na busca por informações climáticas, tanto por parte do governo quanto pela população. Apesar deste crescimento, é necessária uma melhora na forma como a informação é repassada para a população em geral, ou seja, para usuários leigos que precisam de informações claras e acessíveis. 
No Brasil existem agências responsáveis por repassar informações climáticas para população e para os órgãos de controle de desastres ambientais. Em Pernambuco, a empresa responsável pela previsão do tempo é a Agência Pernambucana de Águas e Climas (APAC). A APAC também é responsável por executar a Política Estadual de Recursos Hídricos, além de planejar e disciplinar os usos múltiplos da água em âmbito estadual. No que diz respeito à população, as buscas por essas informações são motivadas por diversos fins, porém, neste artigo é abordado a problemática da incompreensão de termos técnicos pelo público não especializado.

Com o intuito de melhorar a forma como os dados climáticos são apresentados à população, essa pesquisa buscou uma solução tecnológica para facilitar esse processo. Para tal, como abordagem norteadora foram utilizadas técnicas de Gestão do Conhecimento (KM - do inglês Knowledge Management), mais especificamente as etapas do Ciclo KM. O Ciclo KM trata a informação em todas as suas etapas, desde a sua criação até a sua disseminação.

Durante a execução deste trabalho as etapas do Ciclo KM foram realizadas de forma que na captura e criação do conhecimento foram levantados e analisados os problemas propostos afim de encontrar o mais relevante. No compartilhamento e disseminação do conhecimento foi criado um protótipo de uma ferramenta de chatbot que seria disseminado através da plataforma web da instituição e da rede social Telegram ${ }^{1}$. Como última fase foi realizada a etapa de aplicação do conhecimento com apresentação da proposta tecnológica aos interessados (APAC, população).

Sendo assim, o objetivo deste trabalho é apresentar uma solução viável para melhorar a comunicação das informações do tempo repassadas da APAC para a sociedade. Tem-se então a seguinte questão de pesquisa: Como melhorar a comunicação das informações do tempo para o público que não possui conhecimento técnico?

O presente trabalho está dividido nas seguintes seções: na seção 2 será apresentada a fundamentação teórica, na seção 3 será apresentada a execução do Ciclo KM com descrição de suas etapas, na seção 4 será realizada a discussão sobre os resultados, seguido da seção 5 com as conclusões deste trabalho.

\section{Fundamentação Teórica e Metodológica}

De forma geral, as informações são derivadas de dados que sem contexto não possuem significados relevantes. É a partir das informações que o ser humano processa e adquire conhecimento que por sua vez pode ser tácito ou explícito. O conhecimento tácito é resultante dos processos de análise das informações, dos insights subjetivos e argumentos prioritários para processos de inovação e criatividade. $\mathrm{O}$ conhecimento explícito é considerado aquele que pode ser formalizado, sistematizado e facilmente comunicável [Behr \& Nascimento, 2008].

Durante essa pesquisa foram utilizadas as etapas do Ciclo KM que envolvem: Captura que refere-se à identificação e posterior codificação do conhecimento interno e know-how da organização e/ou conhecimento a partir do ambiente externo; Compartilhamento e Disseminação que a partir de uma avaliação do conhecimento

\footnotetext{
${ }^{1}$ https://telegram.org/
} 
capturado promove a contextualização do conteúdo, ou seja, é uma ligação entre o conhecimento e os seus detentores; e Aquisição e Aplicação que após a validação e avaliação do conhecimento como relevante insere-o no repositório de práticas das ações pessoais e organizacionais [Dalkir, 2005]. A figura 1 apresenta o ciclo KM, cuja proposta é sua utilização para aperfeiçoamentos de processos organizacionais.

Para Davenport e Marchand (2004) o conhecimento é essencial dentro de uma empresa, além de ser inteiramente dependente do ser humano, que é produtor e consumidor dele. Pereira (2015) propôs diretrizes para a elaboração de um plano institucional de gestão do conhecimento para o Instituto Nacional de Pesquisas Espaciais (INPE), identificando as melhores práticas a serem aplicadas. Os resultados da pesquisa mostraram que primeiramente deve-se estar focado em reter $o$ conhecimento das pessoas que estão prestes a se aposentar e aplicar este conhecimento na aceleração do aprendizado dos novos ingressantes.

Em Dávila et al. (2014) é possível ver uma análise de GC dentro de uma organização empresarial, onde os autores chegaram à conclusão que o seu uso gerou valor tanto para dentro da empresa como para suas associadas. Já no trabalho Aquino et al. (2018), os autores propõem a utilização do Ciclo KM para tentar solucionar o problema de gestão de informação dentro de uma instituição de ensino. Como conclusão o estudo mostra que a solução proposta viabiliza a padronização e transparência das informações.

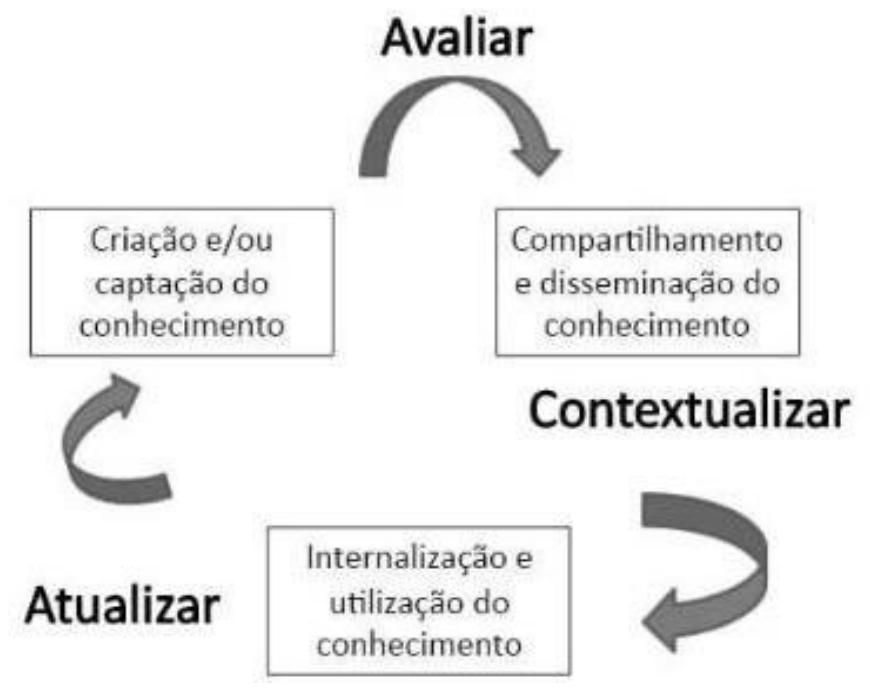

Figura 01. Ciclo KM segundo Dalkir e Liebowitz (2011)

Este trabalho diferencia-se dos demais apresentados por tratar a gestão do conhecimento no contexto climático, apresentando uma solução para uso da população e não de uma organização. Propondo assim a utilização de um chatbot (uma abreviação para robô de chat). O chatbot é um programa de computador que simula uma conversa humana. Dessa forma, automatiza tarefas repetitivas e burocráticas, deixando tempo livre para resolver problemas mais complexos, segundo o Resultados Digitais ${ }^{2}$. Os chatbots já se mostraram muito úteis para resolver problemas, tornando-se indispensáveis para muitas empresas, respondendo a perguntas e interagindo com o público principalmente se há uma grande demanda.

\footnotetext{
${ }^{2}$ https://resultadosdigitais.com.br/blog/chatbot
} 


\section{Execução do Ciclo KM}

Essa pesquisa seguiu uma metodologia proposta pelo ciclo KM que será apresentada nesta seção. Na primeira etapa (captura) foi levantado dados a partir do conhecimento organizacional tácito e explícito para encontrar algum problema relevante. $\mathrm{Na}$ segunda etapa (compartilhamento) foi proposto o desenvolvimento de chatbot para disseminação do conhecimento a partir da interligação de informações do clima/tempo. Por último a etapa de aplicação do conhecimento contou com o desenvolvimento de um cenário que simula a interação de um humano com um chatbot. A tabela 01 mostra as ferramentas, e os respectivos propósitos, que auxiliam a execução das etapas do ciclo KM neste trabalho.

Tabela 01. Etapas do ciclo KM

\begin{tabular}{|l|l|l|}
\hline \multicolumn{1}{|c|}{ Etapa } & \multicolumn{1}{|c|}{ Ferramenta } & \multicolumn{1}{c|}{ Propósito } \\
\hline \multirow{1}{*}{$\begin{array}{l}\text { Captura do } \\
\text { conhecimento }\end{array}$} & Pesquisa desk & $\begin{array}{l}\text { Analisar o site da organização-alvo para } \\
\text { entendimento do problema. }\end{array}$ \\
\cline { 2 - 3 } & Pesquisa de campo & $\begin{array}{l}\text { Discutir e entender como os trabalhos são } \\
\text { realizados na organização-alvo. } \\
\text { Diagnosticar a situação e problemas mais } \\
\text { recorrentes. }\end{array}$ \\
\cline { 2 - 3 } & Pesquisa exploratória & $\begin{array}{l}\text { Comparação dos diferentes meios de } \\
\text { comunicação do clima. }\end{array}$ \\
\cline { 2 - 3 } & Mapa mental & $\begin{array}{l}\text { Estruturar o organograma da organização } \\
\text { para identificar as relações entre os } \\
\text { setores. }\end{array}$ \\
\cline { 2 - 3 } & Storytelling & $\begin{array}{l}\text { Conscientizar sobre os benefícios da } \\
\text { implantação de um processo KM. }\end{array}$ \\
\hline $\begin{array}{l}\text { Compartilhamento } \\
\text { e disseminação do } \\
\text { conhecimento }\end{array}$ & Chatbot & $\begin{array}{l}\text { Ferramenta para comunicação do tempo. } \\
\text { Extrair a informação diretamente do site } \\
\text { da APAC. }\end{array}$ \\
\hline $\begin{array}{l}\text { Aplicação do } \\
\text { conhecimento }\end{array}$ & Prototipação de cenários & $\begin{array}{l}\text { Descrição de possibilidade de uso do } \\
\text { chatbot pelos usuários. }\end{array}$ \\
\hline
\end{tabular}

\subsection{Captura e Criação do Conhecimento}

O primeiro passo desta etapa foi a realização de uma pesquisa desk de avaliação do site da APAC. Uma pesquisa desk é a pesquisa realizada geralmente na Internet através de computador (desktop) para consulta a documentos digitais. Em seguida foi realizado um brainstorming para discutir os problemas comuns de gestão do conhecimento enfrentados pela empresa. Além disto, foram realizadas observações e entrevistas com os profissionais afim de levantar dados em relação ao compartilhamento de boletins meteorológico.

Para facilitar a compreensão dos dados, foi construído um mapa mental, que pode ser visto na Figura 1, com a síntese de fatores que poderiam ser explorados durante a pesquisa. De acordo com Cardoso, Yamaguchi e Vieira (2016), o mapa mental pode 
ser considerado uma ferramenta para organizar ideias por meio de palavra-chave, cores e imagens em uma estrutura que se expande a partir de um ponto discursivo principal. Os mapas mentais favorecem o aprendizado e, consequentemente, melhoram a produtividade pessoal. A análise do mapa mental permitiu notar que existe uma grande deficiência no repasse das informações meteorológicas fornecidas pela APAC uma vez que no website as informações sobre o tempo agora são fornecidas de forma técnica e podem ser de difícil compreensão para o usuário não especializado.

$\mathrm{Na}$ Tabela 02 pode ser visto o resultado da pesquisa realizada, onde encontra-se o comparativo entre sites de quatro organizações que fazem o monitoramento do tempo/clima. Os sites escolhidos tratam-se de dois órgãos estaduais e dois nacionais. A APAC o órgão meteorológico no estado de Pernambuco, a FUNCEME (Fundação Cearense de Meteorologia e Recursos Hídricos) que é uma das referências em meteorologia no Nordeste, a CPTEC (Centro de Previsão de Tempo e Estudos Climáticos) e a INMET (Instituto Nacional de Meteorologia) que são órgãos nacionais de meteorologia.

Os critérios utilizados para fins de comparação foram: (1) Elementos visuais na exibição do tempo, verifica por exemplo, se o website possui figuras ilustrativas; (2) Fácil acesso ao monitoramento do tempo, ou seja, facilidade em encontrar as informações; (3) Informações de fácil entendimento, analisando se as informações fornecidas são compreendidas pelo usuário não especializado; (4) Informações detalhadas, ou seja, o grau de detalhamento do que está ocorrendo e irá ocorrer.

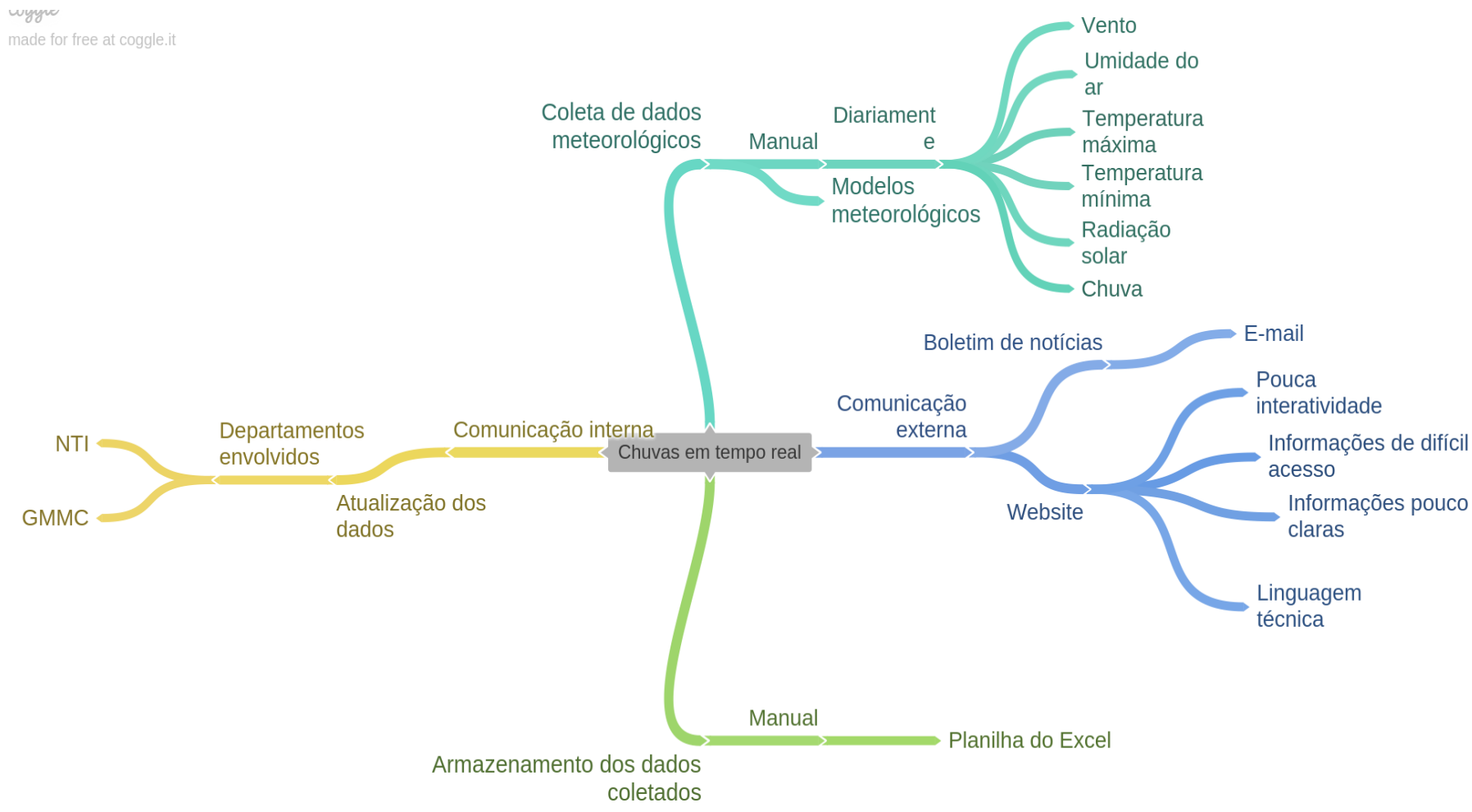

Figura 02. Mapa Mental 
Tabela 02. Comparação entre as principais agências para monitoramento climático.

\begin{tabular}{|l|l|l|l|l|}
\hline \multicolumn{1}{|c|}{ Atributos de valor } & \multicolumn{1}{|c|}{ APAC } & CPTEC & \multicolumn{1}{|c|}{ FUNCEME } & \multicolumn{1}{|c|}{ INMET } \\
\hline $\begin{array}{l}\text { Elementos visuais na exibição do } \\
\text { tempo }\end{array}$ & Não & Não & Não & Não \\
\hline $\begin{array}{l}\text { Fácil acesso ao monitoramento } \\
\text { do tempo }\end{array}$ & Não & Não & Sim & Sim \\
\hline $\begin{array}{l}\text { Informações de fácil } \\
\text { entendimento Não }\end{array}$ & Não & Não & Não \\
\hline Informações detalhadas & Sim & Não & Sim & Não \\
\hline
\end{tabular}

Após o diagnóstico do cenário de pesquisa optou-se pelo uso de um chatbot interativo, para realizar a comunicação do tempo agora de forma mais eficiente para o usuário. Para validação da ideia junto a APAC, foi criado um storytelling que pode ser encontrado no link ${ }^{3}$.

\subsection{Compartilhamento e Disseminação do Conhecimento}

Como descrito na literatura tempo e clima são questões distintas, para Cavalcanti et al. (2015) clima diz respeito a média das condições do tempo ao longo de um período de algumas décadas, o que sentimos no dia a dia é o sistema de tempo que provoca a variabilidade que afetam a vida humana.

Neste trabalho foi considerado o tempo agora, que seria a variação no momento em que o usuário busca a informação. Para compartilhar essas informações propõe-se a utilização de um chatbot nas plataformas $W e b$ e a mídia social de troca de mensagens, Telegram. Optou-se pelo Telegram por ser possível sua integração com sistemas de chatbot externos.

O funcionamento do chatbot é muito simples, o usuário vai escolher a região onde mora e depois poderá perguntar sobre o tempo agora e receber uma resposta com informações precisas e de fáceis de entendimento para o público não especializado. A solução KM permite ao usuário optar por receber o boletim por e-mail e buscar informações de acordo com a região da cidade que ele escolher, sendo Norte, Sul, Leste ou Oeste.

\subsection{Aplicação do Conhecimento}

A última atividade do ciclo KM (aplicação do conhecimento) consistiu em apresentar a solução, em forma de protótipo aos funcionários da APAC além de um grupo de usuários comuns sem conhecimentos técnicos para obter um feedback.

Foram aplicados dois questionários um para a equipe da APAC e outro para um grupo de pessoas. Este primeiro teve um resultado bastante positivo, porém com algumas sugestões que apontavam para integração com os demais serviços da agência. O segundo questionário mostrou que os usuários tiveram uma maior tendência a aceitar a disponibilização em uma plataforma de rede social, como o caso do Telegram.

\footnotetext{
${ }^{3} \mathrm{http}: / /$ urlshortener.me/bsr95LDs
} 
Um outro cenário também foi simulado com o chatbot na página web da APAC. A ferramenta estará vinculada ao site e o usuário poderá interagir abrindo a caixa de diálogo que ficará ao lado direito da página. A escolha foi baseada na facilidade para acessar a página pelo celular ou página web.

\section{Resultados Obtidos}

Esta seção descreverá os resultados obtidos através dos questionários aplicados primeiro aos funcionários da APAC (Figura 3) e em segundo a um grupo de pessoas (Figura 4). O primeiro questionário contou com seis respostas, vistas abaixo na figura 3 , que serão descritas e listadas a seguir:

1) Avaliar em uma escala de zero (inútil) a cinco (muito útil) o quão útil é o chatbot como ferramenta para comunicação do clima, todas as seis respostas indicaram a nota cinco.

2) Descobrir o quão prático a solução mostrou-se quando utilizada dentro do site da APAC, e sua viabilidade para hospedar o chatbot dentro do próprio site da organização. Cinco dos respondentes classificaram como muito prático, apenas um classificou a praticidade como quatro na escala.

3) Averiguar a praticidade da ferramenta no Telegram. Nesse caso houve uma unanimidade que seria muito prático usar a solução dentro da rede social.

4) Verificar a contribuição da proposta e se a ferramenta seria útil na comunicação do clima. A maior parte dos respondentes acredita que a ferramenta é sim útil para comunicar sobre o clima.

5) Examinar em quais aspectos da organização a ferramenta seria útil tornando possível perceber que a comunicação sobre o clima, alertas sobre chuva forte e canal de comunicação com a população foram os aspectos mais citados.

6) Para última questão foi proposta uma pergunta aberta para propor melhorias na ferramenta. Essas respostas foram sintetizadas na Tabela 3.

A Tabela 03 mostrou que deve haver uma expansão na proposta para abranger outras áreas de atuação da APAC. As respostas mostram que a proposta pode contribuir, inclusive em outras áreas de atuação, porém precisa de ajustes na sua concepção.

Tabela 03. Resposta para a questão 6.

\section{Há algo na ferramenta proposta que você recomendaria uma melhoria?}

Não. A linguagem de comunicação e o fluxo seguido na aplicação parecem bons.

Amadurecer mais a ideia e conversar mais com áreas da APAC para adicionar mais serviços, sobretudo com a comunicação.

Ampliar para as outras áreas da APAC.

Com atenção a questão da linguagem, tendo em vista a minimização de ruídos.

Expandir para outras áreas da APAC, como a gestão de organismos de bacia e outorga e fiscalização; utilizar emoticons para encurtar as mensagens.

No segundo questionário, cuja resposta pode ser vista abaixo na figura 04, aplicado virtualmente, um total de cinco resposta foram obtidas, os objetivos dessas perguntas serão descritos e listados a seguir: 
1) Observar se o usuário vê utilidade na ferramenta para comunicação do clima. Dois dos cinco consideraram-na muito útil, enquanto o restante deu nota baixa.

2) Colher o grau de praticidade que o chatbot poderia oferecer, caso utilizado dentro do site da APAC. Apenas duas pessoas acreditam que esta seria uma abordagem prática a ser utilizada.

3) Verificar a praticidade da ferramenta dentro do aplicativo Telegram, que se provou ter uma maior aceitação.

4) Mensurar se o usuário estaria disposto a utilizar a solução de chatbot, caso a ferramenta estivesse presente dentro do site da APAC. Os participantes mostraram-se divididos quanto às suas respostas, dois afirmaram que com certeza utilizariam, enquanto o restante mostrou um grau inferior de interesse.

5) Medir a aceitação da população quanto a utilizar a ferramenta dentro do Telegram. $\mathrm{O}$ aplicativo teve uma maior aceitação, onde dois deram nota cinto, dois deram nota quatro e um deu nota três.

Diante das avaliações realizadas tanto com os profissionais da APAC quanto os usuários, foi possível notar que a ideia possui um grande potencial sendo necessário ser aprimorada para melhor atender ambos os lados interessados. É necessário que sejam realizados mais estudos e com uma fatia maior da população para melhor refletir a utilidade da solução proposta. Porém, tendo em vista a avaliação positiva por ambos os atores pesquisados verifica-se que a problemática é relevante dentro do campo de pesquisa aplicando computação à gestão do meio ambiente.

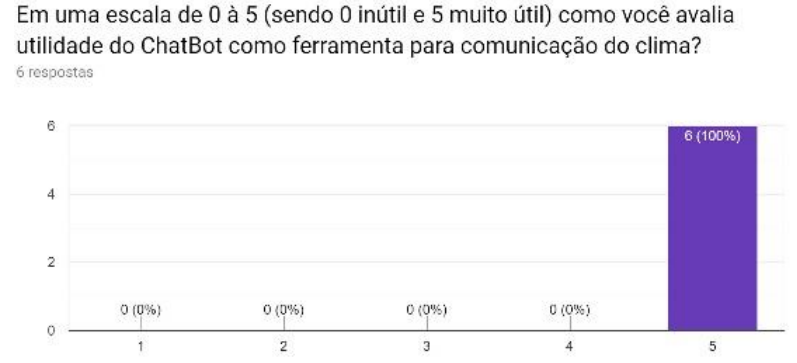

Como você avalia a praticidade da ferramenta dentro do aplicativo Telegram?

6 respostas

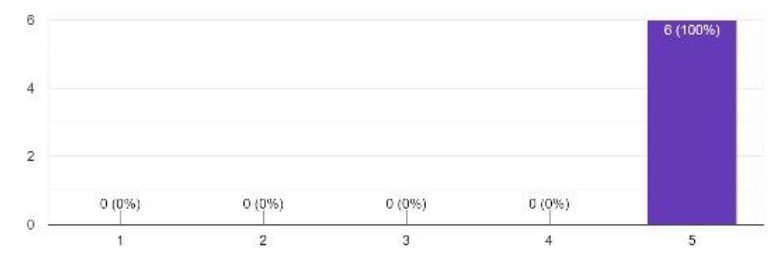

A ferramenta apresentada atende a quais aspectos listados abaixo?

$$
6 \text { respostas }
$$

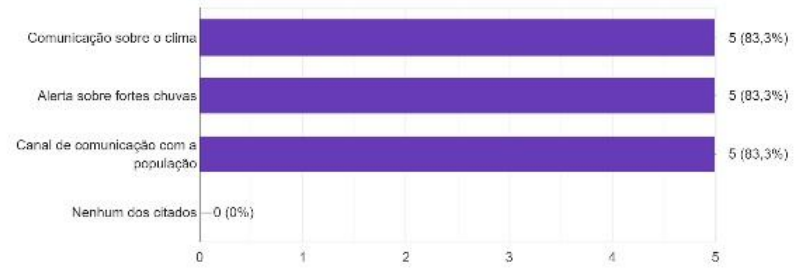

Figura 03. Gráficos de respostas APAC
Como você avalia a praticidade da ferramenta dentro do site da APAC?

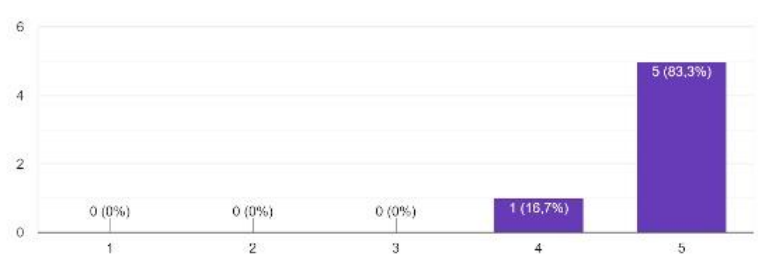

Você acredita que a ferramenta pode ser útil na comunicação do clima?
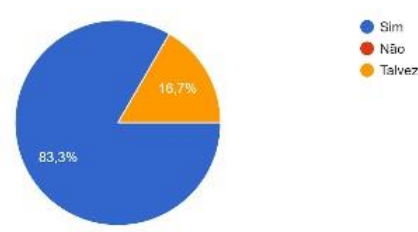
Em uma escala de 0 à 5 (sendo 0 inútil e 5 muito útil) como você avalia utilidade do ChatBot como ferramenta para comunicação do clima? 5 respostas

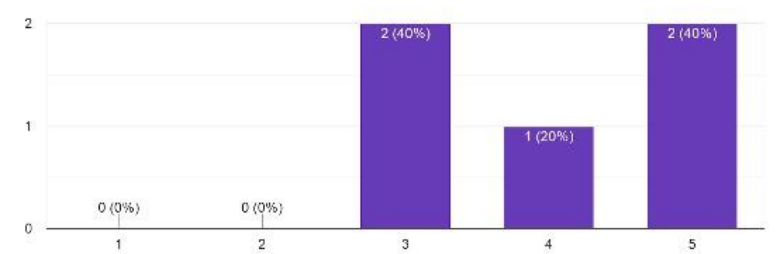

Como você avalia a praticidade da ferramenta dentro do aplicativo Telegram?

5 respostas

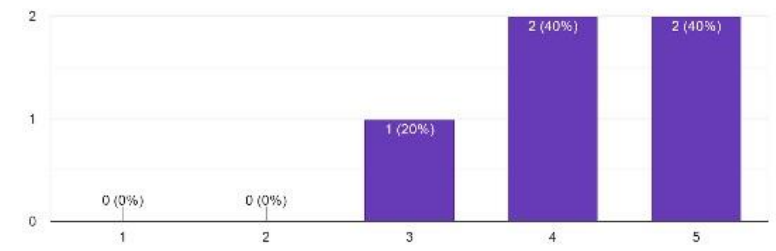

Como você avalia a praticidade da ferramenta dentro do site da APAC?

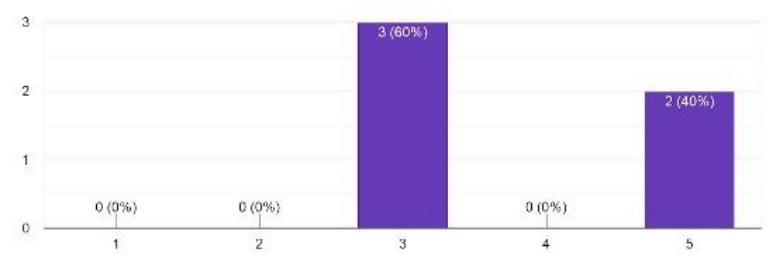

Em uma escala de 0 a 5 quanto você acredita que utilizaria a ferramenta dentro do site da APAC? 5 respostas

Em uma escala de 0 a 5 quanto você acredita que utilizaria a ferramenta dentro do Telegram? 5 respostas
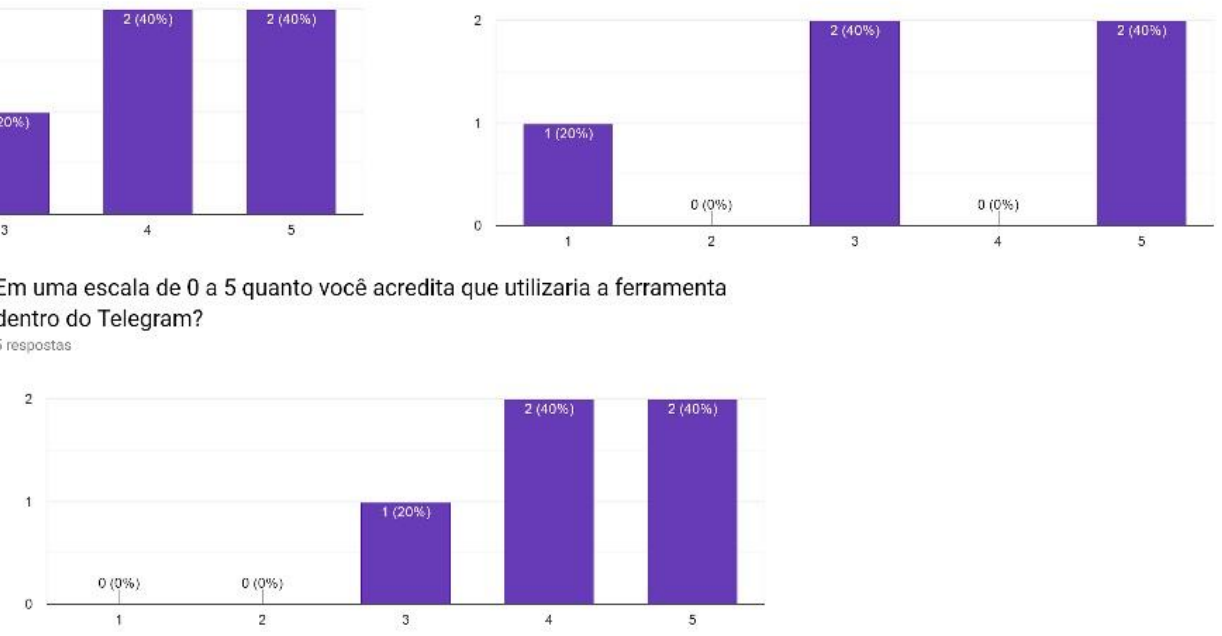

Figura 04. Gráficos de respostas dos usuários

\section{Considerações Finais}

O trabalho desenvolvido procurou trazer uma solução viável para o problema de comunicação do tempo agora para pessoas sem conhecimento técnico, tentando trazer uma forma mais prática e clara para o usuário receber as informações. Foi utilizado o ciclo KM para tratar as informações colhidas durante a pesquisa, e transformá-las em algo mais acessível para a população. Para tentar solucionar o problema uma ferramenta de chatbot foi proposta. Um protótipo como exemplo de uso dentro do site da APAC e da rede social Telegram foi criado. Essa proposta passou por uma validação com os funcionários da APAC e uma pequena amostra de usuários finais, recebendo um feedback positivo de ambas as partes.

Pretendeu-se com isto mitigar o problema de busca de informações por parte da população em meios de comunicação de organizações climáticas. Através disso verificou-se a necessidade de abrangência para outras áreas da organização, fator que se mostrou uma limitação, como apontado pelos entrevistados na APAC. Outra limitação foi a não implementação dentro das plataformas que as apresentadas. Apesar disso, os resultados obtidos foram satisfatórios, a maioria das pessoas avaliaram o chatbot como útil.

Como trabalhos futuros, propõe-se a implantação de novas funcionalidades como possibilidade de interações através de voz. Sugere-se que essa ferramenta seja 
implantada na plataforma web, Telegram e similares, para verificar seu uso em um cenário real possibilitando mensurar seu potencial de comunicação e distribuição das informações do tempo agora para a população.

\section{Referências}

Aquino, C. D., Freitas A. K., Santos, M. G., Souza, R. A., Teixeira, M. and Álvaro, R. (2018). Gestão da informação e do conhecimento: uma proposta de solução KM para campi avançados.

Cardoso, J. V. M., Yamaguchi, C. K. and Vieira, A. C. P. (2016). Mapa mental como ferramenta para potencializar o conhecimento numa cooperativa no sul de Santa Catarina. II Workshop de Administração: Virtualização e Gestão os Desafios das Empresas na Virtualização dos Negócios.

Dalkir, K. and Liebowitz, J.(2011). Knowledge Management in Theory and Practice (2). Cambridge, US: The MIT Press.

Dalkir, K. (2005). Knowledge Management in Theory and Practice. Burlington: Elsevier

Davenport, T. and Marchand, D. (2004). A GC é apenas uma boa gestão da informação?. dominando a gestão da informação. pages 189-194.

Dávila, G. A., Fraga, B. D., Diana, J.B. and Spanhol, F.J. (2014). O Ciclo de Gestão do Conhecimento na prática: um estudo nos núcleos empresariais catarinenses. International Journal of Knowledge Engineering and Management, 3 (7), 43-46.

Globalbot. Chatbots: Entenda de vez como funcionam e como implementar. Disponível em: <https://www.globalbot.com.br/chatbot> Acesso em: 04 de Dezembro de 2018.

Lave, J., and Wenger, E. (1991). Situated learning: Legitimate peripheral participation. Cambridge university press.

Panahi, S., Watson, J., and Partridge, H. (2016). Information encountering on social media and tacit knowledge sharing. Journal of Information Science, 42 (4), 539-550.

Pereira, C. M., Ribeiro, M. L., Siqueira, E. S., and Urbina, L. M. S. (2015). Gestão do conhecimento em uma instituição de pesquisas espaciais: base nos modelos esa e nasa. pages 1-25. Anais do V Congresso Internacional do Conhecimento e Inovação. Florianópolis: UFSC. 